\title{
To Develop Racing Application for Real Time Results Calculation using GPS and GPRS/GSM Device
}

\author{
Poonam Gulab Bhogade \\ Department of Computer Engineering and \\ Information Technology \\ College of Engineering Pune
}

\author{
Vandana S. Inamdar \\ Department of Computer Engineering and \\ Information Technology \\ College of Engineering Pune
}

\begin{abstract}
This paper presents that, integration of GPS and GPRS/GSM device for any kind of vehicle gives an suitable output for real-time racing of vehicles (Bikes, Jet Skies).To develop real time racing application, use of the two technologies is made, i.e. Global System for Mobiles/General Packet Radio Service (GSM/GPRS) which provide $2 \mathrm{G}$ and $3 \mathrm{G}$ technologies and satellite-based navigation system consisting of several satellites revolving around the earth i.e. Global Positioning System. The GPS and GSM/GPRS device will provide the solution for tracing of path and for real time racing of vehicles (Bikes, Jet skies). A real-time vehicle tracking system proposes the use of a GPS technology module to receive the location of the vehicle, to forward data into microcontroller and connect to internet by a GPRS technology for displaying a real time results on the website map developed by Google map. It allows inspection of vehicles with the Google map on a real-time website. Also vehicles can be monitored and located very effectively and results can be declared faster. GPS has various applications such as in military projects, also used as a tool in commerce, scientific uses, tracking and surveillance etc.
\end{abstract}

\section{General Terms}

Communication using GPS and GPRS/GSM, Haversine algorithm for distance calculation to get real time results.

\section{Keywords}

Global Positioning System (GPS), General Packet Radio Service/Global System for Mobiles (GPRS/GSM), Advanced Vehicle Locator (AVL).

\section{INTRODUCTION}

Nowadays, vehicles are essential to transport products or goods in many organizations or firms, but there are many problems of using the vehicles for their business such as the delay of the deliveries, driving out of paths, or even stealing oil and products; Also during racing events of vehicles, there is need to keep race data as a backup on server and can be called upon on demand in case of appeals, or used to cross check the actual track of the participants and to consolidate decisions. Therefore, GPS and GPRS/GSM are important technologies for monitoring such problems. GSM is the $2^{\text {nd }} / 3$ rd generation digital cellular network [8]. It is widely deployed around the world. Although improvement to GSM such as the next generation systems have been rolled out to cater for faster data centric traffic and backward compatibility to GSM is still maintained. Navigation Satellite Timing and Ranging Global Positioning System' (NAVSTAR) is also termed as GPS. NAVSTAR gives instantaneous location, speed and time information in all kinds of atmospheric condition. GPS Navigation system is widely adopted in vehicles today [6]. Space based satellite navigation system i.e. GPS consist of four or more GPS satellites. A United States government organization has maintained the GPS and is freely usable to everyone with a GPS receiver/device. Only authorised users having the full accuracy of the service is limited by the GPS. Normally it is the feature of GPS and it also gives protection from spoofing.

GPS divided into 3 major system segments [7]

1. Space segment

2. Control Segment

3. User Segment

Nominal constellation of 24 NAVSTAR satellites constitutes to space segment. Radio frequency (RF) codes and a navigation data messages are broadcasted from each satellite. Monitoring of networks and control facilities are provided by control segment. Management of the satellite constellation and updating the satellite navigation data messages is also done by control segment. Space segment is the satellite constellation in space which is maintained by control segment. Signal from the space segment is given to user segment, or the user receiver equipment which computes the navigation timing and other functions.

For measuring or maintaining orientation based on the principles of angular momentum, gyroscope device is used. Accelerometer and gyroscope is used for reliability. Calculation of one's position by using a previously determined position is the dead reckoning process. Digital atlas shows maps of different scales [10]. Website owners are useful for incorporation of maps in their own web pages and using mapblast, these maps are later syndicated across most major web. All these terms support GPS. This GPS and GPRS/GSM device is used to track and trace anyone and anything these days.

\section{EXISTING SYSTEM}

\subsection{Multi-Tracking System}

It is next generation web based GPS application. It uses GSM/GPRS and GPS network. It provides real time automatic vehicle location, tracking as well as controlling the vehicle and tracing the paths in which the vehicle or the person travelled generating various forms of reports to assist the executive staff. It also sends alerts/notifications via SMS or Emails.

The components of multi tracking system are-

- AVL (Advance Vehicle Locator) device:-Teltonika FMXXXX or Go safe platinum device. GPS and GSM connectivity terminal is provided in AVL. It has 4 digital inputs, 4 analog inputs, Fuel counter inputs, External battery input, $1 \mathrm{MB}$ internal memory extensible to $4 \mathrm{MB}$, Power supply: 10-30V, 3 status LEDs, GPRS, SMS or RS232 port provide 
configuration update, Support of TCP/IP or UDP/IP protocol, 7500 record storing capacity (upgradeable up to 30000 )

- Socket Listener:-Socket listener is a software component. It listens for incoming connections on particular socket and connects and communicates with the AVL device. It accepts data and forwards it to Parser.

- Parser and Converter:-Parser is a software component. It accepts data from Parser and reads records, parses each record. It converts each information from HEX format to ASCII format. It dumps the data into Database.

- Web application- Web based GUI allowing user to login and use the application for monitoring, tracing, tracking, controlling vehicle \& generating reports etc.

\subsection{Use of GPS in Car}

GPS is also used in cars. GPS in car system shows the current location of the car. It also assists the driver to reach the predefined location and uses a GPS receiver with GPS display. For tracking the distance travelled on a trip and finding out the vehicle mileage and speed, GPS is used. For maintaining record of driving activity GPS is used. Users monitor the usage of their vehicles using these GPS functionalities. The portable GPS device has increase and enhances safety for the people. It is navigational tool i.e. vital to every traveller. This system is good for the driver but by this system the owner of the vehicle can not know the current vehicle details as and when needed, so this automotive navigation system may be useful for the driver but it is not useful for the owner of the vehicle who may want to keep watch on the driver. However this system will not be able to track the vehicles remotely.

\section{BACKGROUND AND RELATED WORK}

In this section, we analyse the reviews of previous papers and also review solution specifically developed for vehicle (Bike, Jet Ski) racing.

Recently, the GPS technology has been presented in many researches. For determination of vehicle speed and position by GPS, a vehicle warning system is useful. For obtaining information about the location of an individual animal or movable object, an object locator system is responsible.

In this GPS localization accuracy classification [1], Nabil M. Drawil proposed that, any GPS system localization accuracy depends heavily on both the technique; i.e.it uses to calculate locations and the measurements conditions in its surrounding. Measurements condition disambiguation and enhanced location accuracy classification are the two steps for the computation of localization accuracy of GPS. To achieve possible highest accuracy, these measures will enable localization system to manage the localization process and resources. Also, to mitigate the impact of inadequate accuracy on the target application, these measures will enable localization system. In GPS/INS, localization measurements are co-related with digital maps and radiolocation measurements which are used to enhance co-operation among different vehicles to correct their uncertain location estimates. Two novel classification algorithms (CBAC algorithm, HCCU strategy) are proposed which are also used for GPS localization.
Assisted Global Positioning System (A-GPS) has been the enabler of many mobile location-based services (LBS) and is the most popular mobile location technology in recent years. Using acquisition and sensitivity assistance from cellular networks, A-GPS receiver, provides higher detection sensitivity for weak signals in the presence of other strong signals by removing strong inter-satellite interference (ISI). However, these autocorrelation-based search techniques often suffer from inter-satellite interference (ISI), when a target GPS signal to detect is much weaker than other incoming GPS signals. The benefit of the proposed fast Multi-Satellite Maximum likelihood (MSML) algorithm is the improved detection performance for weak signals. MSML employs 3 fast computation algorithms proposed by Seung-Hyun Kong [2]. These computation techniques are algebraic approximation of cross-correlation, deterministic estimation of signal amplitudes and carrier phases and search space reduction utilizing relative code phase and doppeler frequency hypotheses for A-GPS in asynchronous cellular network. MSML also uses mathematical model.

Tashfeen B. Karamat and Mohamed M. Atia [3] explained that the relative GPS positioning is used to cancel commonmode errors such as satellite/receiver clock biases and atmospheric effects. The common approach is to use differential GPS (DGPS) carrier-phase measurements to provide centimeter-meter level accuracy. The tracking model contains heading information reported by users. The extended kalman filter (EKF-DD) algorithm performs measurements from the user (or rover) as well as reference (or base). GPS receiver located at a known location are used to form linear combinations which remove the satellite and receivers clock biases and greatly reduces the orbital, inospheric and troposheric errors for short base-lines (the distance between the rover and receiver). The proposed navigation system which uses code phase DGPS that is tightly-coupled with lowcost inertial and speed measurements to provide a reliable, efficient, and low-cost meter level accuracy for land vehicles. The algorithms is compared by analysing their RMS errors in longitude, latitude and altitude parameters during three real road trajectories which were carried out in different scenarios ranging from relatively clear sky to challenging downtown environments.

In bounded constrained filtering [4] for GPS/INS, Garry A. Einicke proposed that, the integrated GPS/INS systems is used to estimate motion between GPS data by combining inertial navigation solutions with GPS. Namely looselycoupled and tightly-coupled, these are two types of GPS/INS integration. The GPS receiver and the inertial navigation system (INS) are treated as separate navigation systems, in loosely coupled mode. In tightly coupled mode, the GPS receiver and INS are in combined mode. To constrain the altitude and velocity measurements using a priori information, during GPS outages, the GPS and INS integration system application is used which is a developed solution to exhibits improved performance. It is explained that when a GPS outages occur, a performance benefit can be provided by constraining the filter's inputs. When a power supply or other service is not available, that time GPS outages occur.

Baik Hoh and Hui Xiong [5] proposed that, from anonymous samples or segments, paths are reconstructed and for this the target tracking techniques can be used. From an identifying home to other potentially sensitive locations a vehicle can report the traces to follow which can also be allowed by the target tracking technique. For measuring the privacy gain and the achieved data quality for the proposed solutions compared 
to a baseline random sampling technique, a real world GPS data set is used. To control disclosure of samples to thirdparty applications and receives location updates from all participating users, a disclosure control i.e. uncertainty aware path cloaking algorithm is used which depends on a trust worthy privacy server. This algorithm focuses on the problem of achieving guaranteed anonymity in a locational data set that includes location traces from many users, at the time of maintaining high data accuracy. Target tracking, and home identification, these are two methods to reidentify anonymous location traces and it observes that, to provide privacy guarantees for drivers or cannot achieve high application accuracy requirement because of failure of known privacy algorithms in low-density areas. A road map as a graph comprising a set of road segments is represented by the application. The application then make generation of the congestion map and mapping new GPS samples to road segments.

Pham Hoang Dat and Micheal Drieberg proposed that GPS and GSM modem is used for the development of vehicle tracking system [9].Specifically, the system will utilize GPS to obtain a vehicle's coordinate and transmit it using GSM modem to user's phone through the mobile network. The main hardware components of the system are u-blox NEO-6Q GPS receiver module, u-blox LEON-G100 GSM module and Arduino Uno Microcontroller. The developed vehicle tracking system demonstrates the feasibility of near real-time tracking of vehicles and improved customizability, global operability and cost when compared to existing solutions.

\section{COMPARISON}

This section describes the various literature review works which have been done in past few years on GPS and GPRS/GSM, and how this research is distinguished from previous research work. According to Seung-Hyun Kong [1], Global positioning system location estimates contains some errors, they are as follows:

i) Difference between the actual GPS satellite position and the anticipated position, rises into ephemeris error.

ii) Decrease in the speed of the satellite signal causes spherical errors.

ii) Delay of the signal arrival due to its reflection off building surfaces in the area causes multipath error.

Seung-Hyun Kong [1] have investigated that the GPS receiver measurements and the error in its location estimation has relationship.

In fast multi-satellite maximum likelihood acquisition for assisted-GPS [2], the auto correlation-based search techniques often suffer from inter-satellite interference (ISI), when a target GPS signal to detect is much weaker than other incoming GPS signals. There have been a few studies on the simultaneous acquisition for multi-satellite (MS) to improve the detection sensitivity for weak GPS signals introduced in literature.

In achieving guaranteed anonymity in GPS traces via uncertainty-aware path cloaking [5], using real-world GPS data set, we measure the privacy gain and the achieved data quality. Most drivers shared same workplace, so it creates confusion. Also there is limitation of data set. We conclude that, a probe-vehicle-based automotive traffic monitoring system supports the estimation of traffic congestion from GPS velocity measurements which is reported from many drivers. The commercial land vehicles, unmanned autonomous vehicles, personal location and navigation uses mainly the low-cost navigation solution which is provided by performance enhancement of MEMS-based INS/GPS integration.

NAVSTAR GPS [7] user equipment introduction provides GPS platform to track vehicles but not suitable for fast moving vehicles due to GPS lock problem. When there is no satellite is visible, then it creates GPS lock problem. GPS lock problem result into loss of data.

Pham Hoang Dat [9] explained that, the system is able to obtain a vehicle's GPS coordinate and transmit it using the GSM modem to user's phone. The developed vehicle tracking system demonstrates the feasibility of near real-time tracking of vehicles, which can be used for security of personal vehicle, public transportation systems, fleet management and many other applications.

However, no papers have demonstrated the GPS and GPRS technologies for a real-time vehicle tracking based on the Google map. Existing system was a manual work. They used to declare results after some time. Those results were having manual errors. So In this paper we have given solution to this problem by developing the system which will calculate the real time results using GPS and GSM data.

\section{CONCLUSION AND FUTURE SCOPE}

GPS and GSM integration for vehicle and other objects tracking can be very helpful instead of using GPS network alone. This paper develops the real time result calculation of races. We used concepts of GPS and GSM to get the required data. We used interpolation distance formula for earth, triangulation method and Haversine algorithms for distance calculation to get real time results. The extensive performance evaluation and optimization of the overall system, especially in terms of accuracy and precision of position estimation is necessary. For tracking and tracing of multiple movable objects at a same time is possible by using GPS and GPRS/GSM device. Using this GPS and GPRS/GSM device we can directly organise any event of racing. This system can be further extended for multiple applications to achieve faster and accurate results. Future work will also focus on more accuracy and precision of position estimation to get more accurate real time results.

\section{ACKNOWLEDGMENTS}

We would like to acknowledge my vigorous thanks to my guide for giving valuable suggestions which helped me a lot in my research work and I also want to thank my friends for helping me in this research work by giving me there feedback on my research work.

\section{REFERENCES}

[1] Seung-Hyun Kong, Member, IEEE "fast multi-satellite ML acquisition for A-GPS" IEEE transaction on Wireless Communications, vol. 13, no. 9, September 2014.

[2] Nabil M. Drawil, Member, IEEE, Haitham M. Amar, Member, IEEE, and Otman A. Basir, Member, IEEE "GPS localization accuracy classification: A contextbased approach" IEEE transaction on Intelligent Transportation Systems, vol. 14, no. 1, March 2013.

[3] Tashfeen B. Karamat, Member, IEEE, Mohamed M. Atia, Member, IEEE, and Aboelmagd Noureldin, Senior Member, IEEE "Performance analysis of code-phasebased relative GPS positioning and its integration with 
land vehicle's motion sensors" IEEE sensors journal, vol. 14, no. 9, September 2014

[4] Garry A. Einicke, Senior Member, IEEE, Gianluca Falco, and John T. Malos "Bounded constrained filtering for GPS/INS integration" IEEE transaction on Automatic Control, vol. 58, no. 1, January 2013.

[5] Baik Hoh, Marco Gruteser, Hui Xiong, Senior Member, IEEE, and Ansaf Alrabady "Achieving guaranteed anonymity in GPS traces via uncertainty-aware path cloaking" IEEE transaction on Mobile Computing, vol. 9 , no. 8, August 2010.

[6] A. EI-Rabbany, Introduction to GPS: The Global Positioning System, Norwood, MA: Artech House, 2006.

[7] D. K. Elliott and H. Christopher, Understanding GPS Principles and Application, Norwood, MA: Artech House, 2006.
[8] G. Gu and G. Peng, "The survey of GSM wireless communication system," in Proc. International Conference on Computer and Information Application (ICCIA), pp.121,124, Dec. 2010

[9] Pham Hoang Dat, Micheal Drieberg and Nguyen Chi Cuong Electrical and Electronics Engineering Department, Universiti Teknologi PETRONAS, Malaysia "Development of Vehicle Tracking System Using GPS \& GSM Modem" 2013 IEEE Conference on Open System s(ICOS), December 2-4, 2013, Sarawak, Malaysia.

[10] El-Medany,W.;Al-Omary,A.;Al-Hakim,R.;Al Irhayim, S.; Nusaif,M.,"A Cost Effective Real-Time Tracking System Prototype Using Integrated GPS/GPRS Module," Wireless and Mobile Communications (ICWMC),2010 6th International Conference on,vol.,no.,pp.521,525,2025Sept.2010. 